Comp. Mater. Sci., Vol. 96, pp. 102-107, (2015) doi:10.1016/j.commatsci.2014.08.047

\title{
Modelling the Microstructure of Martensitic Steels
}

\author{
A. Rahnama \\ Department of Materials, Imperial College London \\ Exhibition road, London, SW7 2AZ, England \\ R.S. Qin, \\ Department of Materials, Imperial College London \\ Exhibition road, London, SW7 2AZ, England
}

April 27, 2015

\begin{abstract}
A method based on the kinetics of crystal growth has been developed and applied to the computation of three-dimensional microstrucuture in austenite-matensite steels. The detailed crystallography of the transformation is used to model a realistic martensitic microstrucuture during the transformation without an external system of stresses. The interaction energy based on the plastic work model is taken into account to compute the variant selection in an austenitic stainless steel and formation of martensite under externally applied stress.
\end{abstract}

Keywords: Martensitic transformation, three-dimensional microstructure, crystallography, variant selection.

\section{Introduction}

Martensitic Transformation in steels normally occurs in an athermal manner, during cooling in a temperature range that can be accurately determined for different steels. When the martensitic transformation occurs, the austenite transforms to martensite through a shape change which is an invariant plane-strain (IPS) [1]. The rate of transformation can reach the speed of sound in metals. Martensite forms in 24 crystallographic variants in each austenite grain. The chemical driving force $\Delta G$ which depends on the composition and transformation temperature applies to all the variants equally. Generally, each variant has an equal chance of existence. However, since martensitic transformation is a deformation, an externally applied stress will favour those variants that comply with the stress and as a result variant selection occurs [2].

Consider an austenite grain with sample axes which are defined by an orthonormal set of basis vectors $\left[\gamma ; a_{1}\right],\left[\gamma, a_{2}\right]$, and $\left[\gamma, a_{3}\right]$. The matrix notation used here is due to 
Bowles and MacKenzie [27].The real basis is referred to as ' $\gamma$ ' and its corresponding reciprocal basis is defined using the basis symbol ' $\gamma^{*}$. The IPS can be represented by a $3 \times 3$ matrix $(\gamma \mathrm{P} \gamma)$ such that $[1,3,4]$ :

$$
(\gamma \mathrm{P} \gamma)=\left(\begin{array}{ccc}
1+m d_{1} p_{1} & m d_{1} p_{2} & m d_{1} p_{3} \\
m d_{2} p_{1} & 1+m d_{2} p_{2} & m d_{2} p_{3} \\
m d_{3} p_{1} & m d_{3} p_{2} & 1+m d_{3} p_{3}
\end{array}\right)
$$

where $[\gamma ; \mathbf{d}]=\left[d_{1}, d_{2}, d_{3}\right]$ are the components of $\mathbf{d}$ in the $\gamma$ basis, a unit vector which points toward the direction of the displacement. $\left[\mathbf{p}, \gamma^{*}\right]$ are the component of the unit invariant-plane normal in the $\gamma^{*}$ basis (normal to the habit plane). $m$ is the magnitude of the shape deformation.

The variant selection occurs if the transformation develops under the externally applied system of stresses. The interactions between martensite and these stresses affects the mechanical free energy of the individual variants depending on their crystallographic orientation and will produce an energy which adds to the chemical driving force. Variant selection occurs when the interaction energy is large enough compared to the chemical free energy $\Delta G[5,6]$.

Different approaches have been used to calculate the interaction energy $U$ between applied stress and the transformation strain. Humbert et al. [6] used a method based on the elasticity theory rather than the plastic model of Patel and Cohen [2]. However, since the transformation strain is plastic, the plastic work explained in Ref [2] gives the correct value of interaction energy [5]. According to Patel and Cohen, the interaction energy between the applied stress and martensitic transformation can be simply described as

$$
U=\sigma_{N} \times \delta+\tau \times s
$$

where $\sigma_{N}$ and $\tau$ are the normal component of stress and corresponding resolved shear stress on the habit plane in the shear direction, $\delta$ and $s$ are the dilatational and shear strains due to martensitic transformation, respectively. When the normal stress is tensile, $\sigma$ is positive, while the compressive stress makes its numerical value negative. The shear component of the stress is always positive during uniaxial loading [2]. This implies that shear stresses will always aid the transformation while the normal stress may stimulate the martensitic transformation if it is tensile, or oppose it in the case that this component of stress is compressive. Kundu et al. $[5,7,8]$ used the combination of crystallographic theory and interaction energy in their calculation and reliably predicted the overall texture due to martensitic transformation.

In the last few decades, the phase field modelling has been used as a powerful computational method for predicting morphological and microstructural evolution in martensitic phase transformation. Different models have been put forward by various groups of scientists, for instance, Falk [9] proposed a one dimensional model for martensitic phase transformation and exploited the shear strain as the order parameter, Barsch and Krumhansl [10,11] derived governing equation for proper martensitic phase transformation through Ginzburg-Landau theory, Saxena et al. [12] and Rasmussen et al. [13] worked with dimensionless and scaled local deviatoric strains as 
order parameter, Ahluwalia et al. [14, 15] introduced a polycrystal model based on the continuum elasticity, Cui et al. [16] proposed a two dimensional model for generic hexagonal to orthorhombic phase transformation, Shchyglo et al. [17] suggested a systematic way to construct the Landau free energy function in $\mathrm{NiTi}$ and $\mathrm{NiTiCu}$ shape memory alloys, Wang and Khachaturyan [18] proposed a realistic three-dimensional phase field simulation for the generic improper cubic to tetragonal transformation in a single constrained crystal, Li et al. [19] presented a model to predict the precipitation of rhombohedral in a cubic matrix in $\mathrm{Ti}_{11} \mathrm{Ni14}$, Artemev et al. [21] studied the effect of external stress on martensitic phase transformation and shown that external stresses increase the production of those variants which are favoured by applied stresses, Artemev et al. [20] also suggested a model for proper martensitic transformation and simulated two different types of cubic to tetragonal transformation, Jin et al. [22] presented a phase field model for the cubic to trigonal transformation in AuCd alloy, Yamanaka et al. [23] suggested an elastoplastic model to simulate cubic to tetragonal transformation for an elastic perfectly plastic material. In the last years, Levitas et al. developed the Ginzburg-Landau theory for proper martensitic phase transformation in various aspects. They proposed their model in three papers: in the first paper [24], 2-3-4 polynomial for thermal part of Gibbs energy was used and the transformation strain was coupled with the order parameter through 2-3-4 polynomial or quadratic; in the second paper [25], the austenite-martensite Landau model was developed to cover martensite-martensite transformation; and in the third paper [26], it is shown that the 2-3-4-5 polynomial is not the only Landau potential that could be used and alternative Landau potentials were introduced, 2-4-6 polynomial in the Cartesian coordinate system, and two potentials in the hyperspherical coordinate system. All these researches among others have revealed the huge capabilities of phase field modelling in predicting the microstructure evolutions at mesoscale. However, this method could be sometimes mathematically cumbersome. Therefore, the need for fast computational methods for the simulation of the microstructure evolution is becoming important.

In the present work, mathematical models have been programmed to simulate the martensitic transformation. The microstructure of martensite is, firstly, computed when no external stress is applied. For this, a set of crystallography data is deduced using the theory of martensite and employed in calculations. Secondly, during the transformation under an external system of stresses, the same method as used in Ref.[5] is employed. A set of crystallographic data for an austenitic stainless steel is used to programme the growth of martensitic variants in an individual austenite grain. This theory is consistent with all the experimentally observed features of the martensitic transformation $[1,3$, $27,28]$. Through the present work, it is assumed that the materials is free from defects. However, the real materials always have defects which play a significant role in the evolution of martensitic microstructure. Also in the present computation, the stress or strain interactions between plates are not accounted for. The presented model can be used for modelling the martensitic transformation in steels as an alternative method for phase filed method. This model is mathematically simpler and is able to compute the microstructure much faster than phase field method. The proposed model benefits from using the phenomenological theory of martensite crystallography which describes the crystallography and shape of martensite plates correctly. 


\section{Crystallography}

Equation 1 can be simplified as:

$$
(\gamma \mathrm{P} \gamma)=I+m[\gamma ; \mathbf{d}]\left(\mathbf{p} ; \gamma^{*}\right)
$$

where $I$ is the identity matrix. There exist 24 different martensite variants in any asutenite grain, and hence resulting in 24 different IPSs. Fig. 1a shows an arbitrary vector $u$ traversing an austenite grain before transformation. $\Delta u$ indicates its intercept with the austenite grain which will transform to martensite. Because of the transformation, the vector $u$ becomes a new vector $v$ as illustrated in Fig. 1b. The components of this new vector can be determined as follows [5]:

$$
v=P \Delta u+(u-\Delta u)
$$

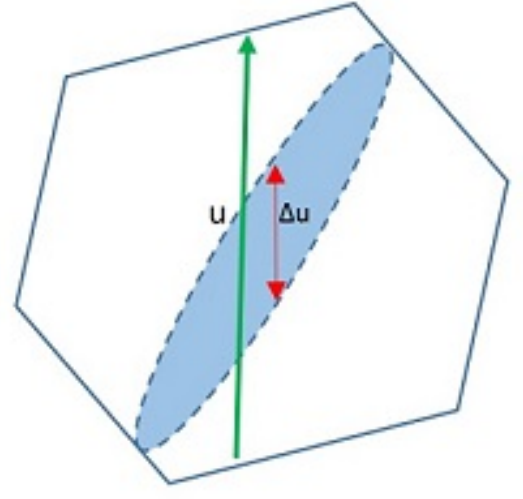

(a)

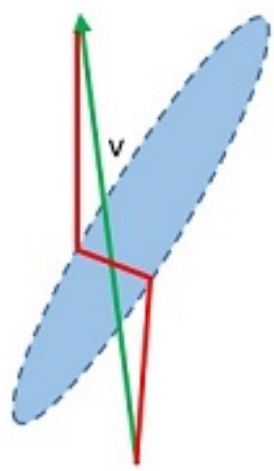

(b)

Figure 1: The formation of an initial vector $u$ due to the formation of martensite. (a) an austenite grain prior to transformation, with the ultimate location of a plate of martensite marked. (b) the following martensite transformation

The change in shape caused by the formation of a particular martensite plate $i$ in an austenite grain, $\left(\gamma \mathrm{P}_{\mathrm{i}} \gamma\right) \equiv \mathrm{P}_{\mathrm{i}}$ is known from the crystallographic theory developed for martensite [3,28]. Knowing this deformation, it is possible to deduce the remaining 23 matrices for a grain of austenite in the sample frame of reference using symmetry operations. Each can be formulated using a similarity transformation as follows:

$$
\left(S \mathrm{P}_{\mathrm{i}} S\right)=(S \mathrm{~J} \gamma)\left(\gamma \mathrm{P}_{\mathrm{i}} \gamma\right)(\gamma \mathrm{J} S)
$$

where the matrix $(S \mathrm{~J} \gamma)$ refer to rotation relating the grain of austenite to the sample axes, and $(\gamma \mathrm{J} S)$ indicates the inverse of this rotation matrix. In such a way, one is able to calculate the components of vector $v$ in the reference frame of the sample. 


\section{Volume fraction of martensite}

\subsection{Transformation from stress free austenite}

The fraction of martensite that forms at any temperature increases with the under cooling below the martensite start temperature. Many researchers have studied the relationship between the martensite volume fraction $f_{\alpha^{\prime}}$ and the quenching temperature. Koistinen and Marburger [29] have proposed an empirical relationship which is called "exponential relationship" as:

$$
f_{\alpha^{\prime}}=1-\exp \left[\beta\left(M_{s}-T\right)\right]
$$

where $\beta$ is approximately equal to a constant of -0.011 for the martensitic transformation in steels of which the carbon weight content is less than $1.1 \%[30,31] . M_{s}$ is the martensite start temperature and $T$ is the quenching temperature in Kelvin. $M_{s}$ can be estimated as follows (concentrations in wt \%)[32]:

$M_{s}\left({ }^{\circ} C\right)=539-423(\% C)-30.4(\% M n)-17.7(\% N i)-12.1(\% C r)-7.5(\% M o)$

This equation is the most widely used equation for determining the martensite fraction below $M_{s}$. They compared their results with that of Harris and Cohen [33] for a variety of steels and have shown that their proposed equation can predict the austenite volume fraction more accurately. However the evolution of the martensite volume fraction is different from the equation above if the austenite grain is plastically deformed prior to transformation [34].

\subsection{Transformation in the presence of applied stress}

Patel and Cohen [2] studied the effect of applied stress on the temperature $\left(M_{s}\right)$. They considered transformation under three different conditions: uniaxial tension, uniaxial compression, and hydrostatic pressure and concluded that external stress contributes algebraically to the free energy change altering the $M_{s}$ temperature. Their results are summarized in Table. 1.

\begin{tabular}{c|ccc}
\hline Stress system & Uniaxial tension & Uniaxial compression & Hydrostatic pressure \\
\hline$\frac{d M_{s}}{d \sigma}$ & $+0.15^{\circ} \mathrm{K} / 1 \mathrm{MPa}$ & $+0.10^{\circ} \mathrm{K} / 1 \mathrm{MPa}$ & $-0.05^{\circ} \mathrm{K} / 1 \mathrm{MPa}$ \\
\hline
\end{tabular}

Table 1: Effect of applied stress on the $M_{s}$ temperature after Patel and Cohen [2].

Interestingly, $M_{s}$ is raised by compression, since the shear strain is much larger than dilatational strain and contributes more effectively than the (negative) compressive normal component which opposes it. In tension, both shear and (positive) normal components of stress aids the transformation and thus the temperature $M_{s}$ is raised even more. $M_{s}$ is lowered by hydrostatic pressure, since this stress system opposes the transformation. All these three conditions of applied systems of stresses are included in the computer program. However, the results only for an austenitic stainless steel are 
presented in the section 5 due to the lack of a complete set of data. The role of the interaction energy and the procedure to calculate the normal and shear components of the external stress are explained in section 5.2 where the numerical results for variant selection are presented. The volume fraction of martensite is used in this computation as a stopping condition.

\section{Nucleation Rate}

\subsection{Isothermal transformation}

Pati and Cohen used quantitative metallographic techniques to evaluate nucleation rates during isothermal martensitic transformation [35]. They measured the nucleation rate directly from calculations of the number of martensitic plates per unit test volume $\left(N_{v}\right)$ and the mean volume plates $(\bar{v})$ and proposed the following equation:

$$
\dot{N}=\frac{d N_{v}}{d t} \frac{1}{1-f}
$$

where $f$ is the volume fraction of martensite and can be easily determined by counting the number of lattice points which are occupied by martensite plates. $\frac{d N_{v}}{d t}$ is the slope of the plot of $N_{v}$ as a function of transformation time. Therefore, having the plot of $\left(N_{v}\right)$ against transformation time for any steel, the nucleation rate at any instant can be obtained. As it is well-known, the nucleation rate increases considerably during the initial part of the transformation due to autocatalysis and then reduces during later stages. Fig. 2 shows the the growth of a few martensite plates and autocatalysis phenomenon at the beginning of the transformation (after 100 time steps). The number of added nucleus is, then, decreased during subsequent time steps as the transformation progresses.

\subsection{Continuous cooling}

Considering continuous nucleation, the number of new martensite phase per unit volume at the temperature $T$ can be determined as [36]:

$$
N=\frac{d N}{d T}\left(M_{s}-T\right)
$$

Nucleation rate, $\frac{d N}{d T}$, can be approximated during continuous cooling for a certain cooling rate. It follows:

$$
\frac{d N}{d T}=\frac{\beta}{\bar{V}}
$$

where $\beta$ is the parameter in the Koistinen and Marburger equation as discussed above. $\bar{V}$ is the mean volume of martensite plates. The computer program is, then, able to calculate the nucleation rate both for isothermal and continuous cooling transformations. 


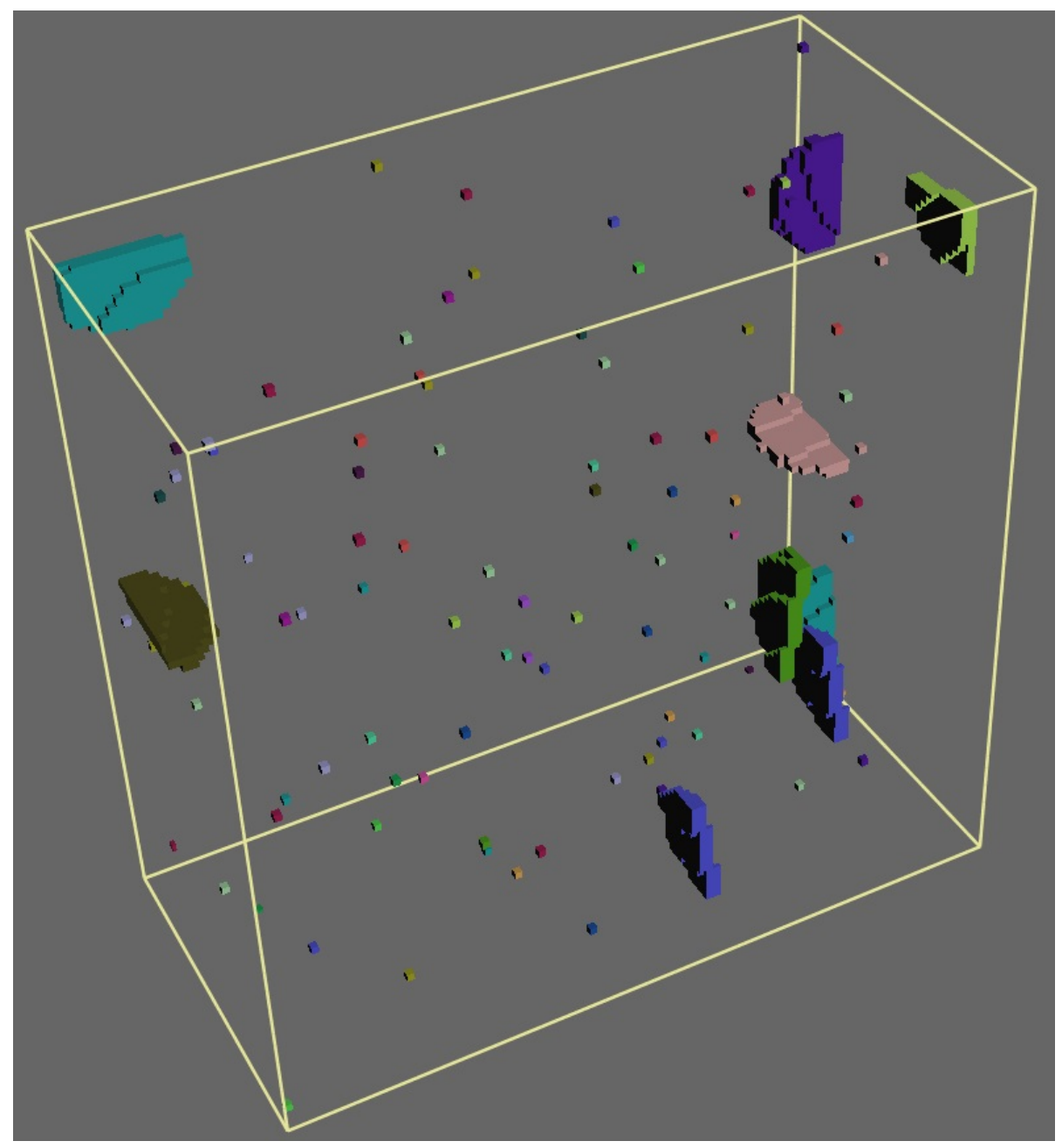

Figure 2: The increase in the nucleation rate during initial part of transformation (autocatalysis).

\section{Results and Discussion}

\subsection{Three dimensional microstructure}

Crystal growth by means of reconstructive transformation obeys a scaling law. The scaling factor can be derived from the Ficks law. The growth of the martensite phase obeys a scaling law which differs from diffusion-controlled transformations due to the displacive nature of the transformation. An ellipsoidal martensite plate forms with various growth velocities along the different axial direction, i.e., $v=v_{x}=v_{y}=\frac{v_{z}}{\eta}$ $[1,36]$. It follows: 


$$
\overrightarrow{v_{\alpha}}=v_{s} \hat{x}+v_{s} \hat{y}+\eta v_{s} \hat{z}
$$

where $v_{s}$ is the speed of sound in steels and $\eta \simeq \frac{\delta}{s}=0.05$ [1]. $\delta$ is the uniaxial dilatation. $s$ is the shear strain due to martensitic transformation. Hence, the grain size is related to the growth time in the format:

$$
d_{\alpha}=\lambda_{\alpha}(\hat{n}) t
$$

where the coefficient $\lambda_{\alpha}(\hat{n})$ is anisotropic. The homogeneous nucleation can be simulated by random selection of growth centres. The location of growth centres in heterogeneous nucleation can be simulated by multiplying a random function with a weight function. The number of growth centres is calculated according to the experimental specified mean grain size. For example, a computational logic frame with volume $L_{x} L_{y} L_{z}$ should contain $L_{x} L_{y} L_{z} / d^{3}$ growth centres.

Equation 11 is defined in the coordinates fixed to an $\alpha$ grain. The $\alpha$ coordinates, the $\gamma$ coordinates, and the sample coordinates are denoted by $\left[\alpha ; v_{\alpha}\right],\left[\gamma ; v_{\alpha}\right]$, and $\left[\right.$ sample $\left.; v_{\alpha}\right]$, respectively. The transformation from one coordinate system to another can be determined according to crystallographic theory explained in section 2 . It follows:

$$
\begin{gathered}
{\left[\gamma ; v_{\alpha}\right]=(\gamma \mathrm{J} \alpha)\left[\alpha ; v_{\alpha}\right]} \\
{\left[\text { sample } ; v_{\alpha}\right]=(\text { sample } \mathrm{J} \gamma)(\gamma \mathrm{J} \alpha)\left[\alpha ; v_{\alpha}\right]}
\end{gathered}
$$

where the transformation $(\gamma \mathrm{J} \alpha)$ is available for many steels. $\alpha$ phase has 24 variants due to cubic symmetry and the coherence/semi-coherence of the interface between $\alpha$ and $\gamma$ phases. When considering a displacive transformation such as martensite the crystallographic set includes shape deformation, habit plane, as well as orientation relationships. This set is mathematically related and should be considered while computing the microstructure of martensite [3,27,28]. The set of data which are used in stress free transformation is summarised in table 2. Martensite plates grow from multiple centre within the grid. The lattice points at the sides and edges of the grid are not part of the domain and are just there to contain the system domain. The growth of a martensite plate is halted by internal boundaries such as grain boundaries (the walls of simulation domain) and collision with another plate, as these are high energy barrier that martensite plates cannot cross or nucleate. This kinetic description of $\alpha$ crystal growth has been programmed by $\mathrm{C}++$ language. The computation of a microstructure represented by $200 \times 200 \times 200$ lattices using an ordinary desktop takes about 20 minutes. The numerical result has been plotted by an in-house visualization software AcaVisual, and the numerical results according to this crystallographic data are shown in Fig. 3. This computer program is applicable to any other scenario provided that habit plane, coordinate transformation matrix $(\gamma \mathrm{J} \alpha)$, and shape deformation matrix $(\gamma \mathrm{J} \gamma)$ are available. Kurdjumov-Sachs or Nishiyama-Wasserman orientation relationships are widely used in the calculation of texture [37]. However, it is now well-known that the true orientation relationships must be irrational in order to lead to the existence of an invariant line between the parent and product lattices. Invariant line is an essential requirement for the martensitic transformation [1, 3, 27, 28]. 


\begin{tabular}{|c|c|c|c|}
\hline habit plane & \multicolumn{3}{|c|}{$\begin{array}{lll}0.197162 & 0.796841 & 0.571115)\end{array}$} \\
\hline coordinate transformation matrix $(\gamma \mathrm{J} \alpha)$ & $\begin{array}{c}0.582598 \\
-0.552752 \\
-0.019285\end{array}$ & $\begin{array}{c}-0.856546 \\
0.576725 \\
-0.134804\end{array}$ & $\left.\begin{array}{c}-0.029884 \\
0.084736 \\
0.791698\end{array}\right)$ \\
\hline Shape deformation matrix $(\gamma \mathrm{P} \gamma)$ & $\begin{array}{c}0.990134 \\
0.032037 \\
-0.028583\end{array}$ & $\begin{array}{c}-0.039875 \\
1.129478 \\
-0.115519\end{array}$ & $\left.\begin{array}{c}-0.028579 \\
0.092800 \\
0.917205\end{array}\right)$ \\
\hline
\end{tabular}

Table 2: Crystallographic data set for stress free martensitic transformation [1].

Fig. 3(a) demonstrates a numerical result of growing $\alpha$ phase within a $\gamma$ grain in homogeneous nucleation. In heterogeneous nucleation where the surface of $\gamma$ grain has a greater chance than that inside the grain, the numerical result is demonstrated in Fig. 3(b). The different colours correspond to the different crystal orientations. The grain morphology of $\alpha$ phase is very similar to that in experimental observation. The crystallographic relationship between $\alpha$ grains and their parent $\gamma$ grain follows theoretical definition precisely. 


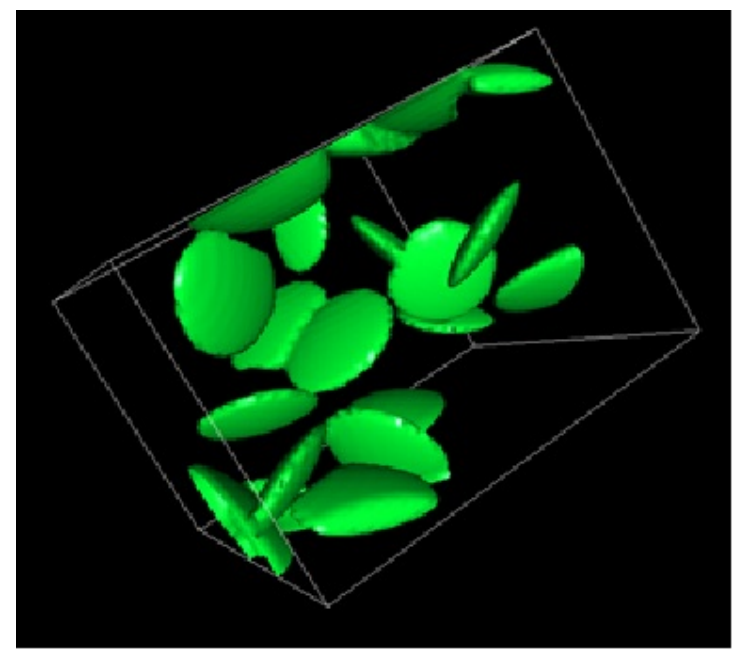

(a)

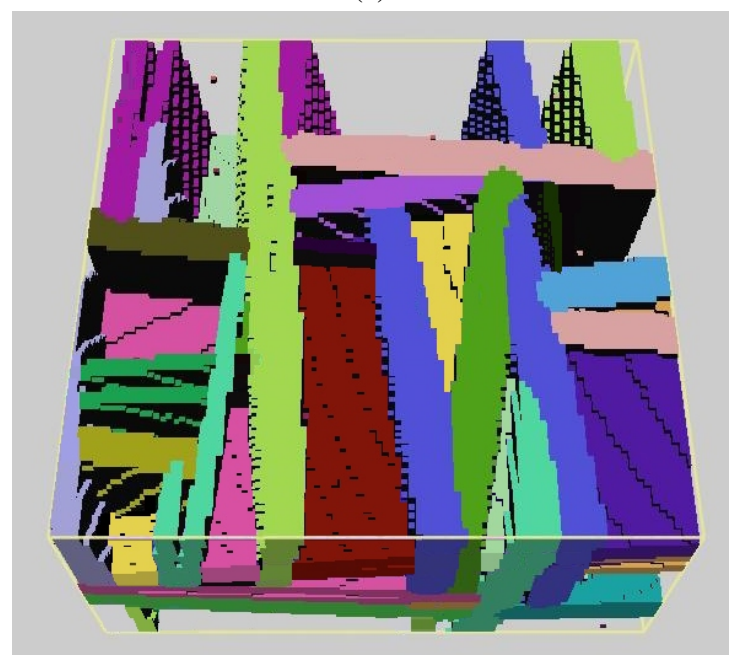

(b)

Figure 3: Numerical results for the growth of $\alpha$ grains within a $\gamma$ grain with (a) homogeneous nucleation and (b) heterogeneous nucleation (each colour represents a crystallographic variant of martensite). The numerical results are for a condition where no external stresses are applied.

The calculated three-dimensional microstructure can be analysed quantitatively. Fig. 4 demonstrates schematically the different types of interfaces. The computer program can determine the volume fraction of $\gamma-\alpha$ interfaces between every two variants of martensite as well as that between martensite and austenite simply through counting the number of lattice points that each interface occupies. 


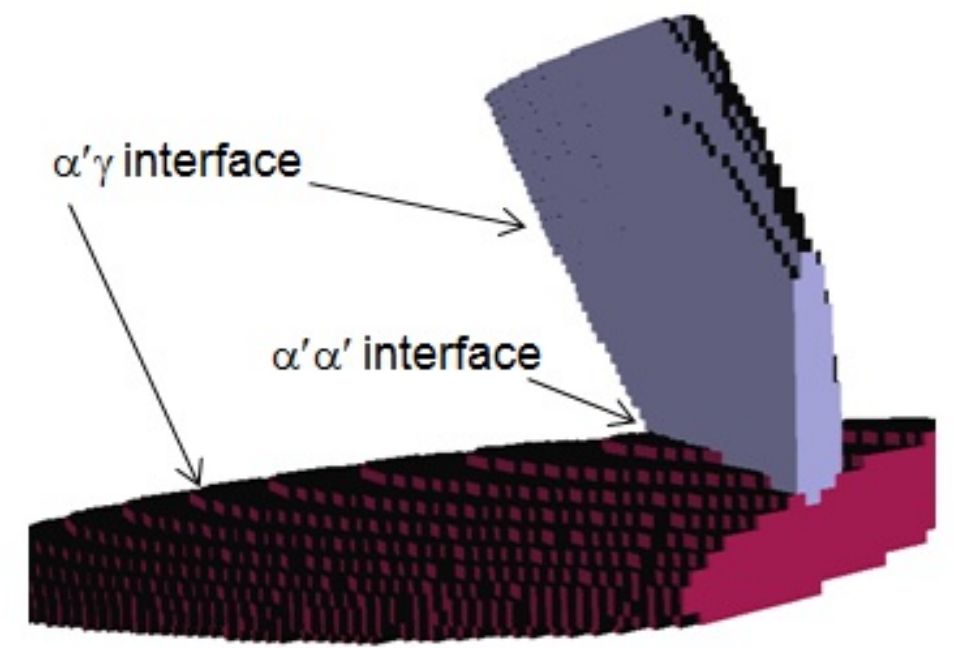

Figure 4: Schematic diagram for information extraction.

\subsection{The variant selection}

Gey et al. [38] in their analysis of an austenitic stainless steel discovered that not all the 24 martensitic variants form during transformation under tensile deformation. They demonstrated the selection of particular variants by comparing the measured pole figure with calculated pole figures for all 24 crystallographic variants of martensite. Humbert et al. [6] calculated the crystallographic texture of an austentic stainless steel transformed into bcc martensite in two stages. They assumed that the austenite first evolved to $\epsilon-$ martensite which eventually transformed into $\alpha^{\prime}$-martensite. However, Kundu et al. [7] have recently reported that in deformed austenitic stainless steels, it is not necessary to calculate the transformation texture in two stages.

They considered that the most favoured crystallographic variants are those with the highest interaction energies.

Using Eq. 2, the value of $U$ can be calculated and compared with the chemical driving force for the martensitic transformation. If the interaction energy of a variant $U \ll \Delta G$, it is unlikely to be selected [7]. The absolute value of the interaction energy, thus, must be calculated precisely, as it plays a very important role in variant selection [39].

Another method for determining the interaction energy is based on elasticity theory as described in Ref. [6]. However the correct procedure for calculating $U$ is the model of Patel and Cohen [2], because the strain associated with martensitic transformation is plastic [7]. Although both methods give the same ranking of $U$, elasticity theory gives a incorrect value for the interaction energy by a factor of 2 [8].

The stress normal to the habit plane can be calculated by resolving the traction stress on the habit plane as follows: 


$$
\sigma_{N}=\sigma_{t} \cdot \mathbf{p}
$$

and the shear stress can be obtained as follows:

$$
\tau=\left(\sigma_{t}-\sigma_{N}\right) \cdot \mathbf{e}
$$

where $\mathbf{e}$ is the unit vector in the direction of shear and the traction stress for a given system of stresses can be shown as:

$$
\sigma_{t}=\left(\begin{array}{lll}
\sigma_{11} & \sigma_{12} & \sigma_{13} \\
\sigma_{21} & \sigma_{22} & \sigma_{23} \\
\sigma_{31} & \sigma_{32} & \sigma_{33}
\end{array}\right)\left(\begin{array}{c}
P_{1} \\
P_{2} \\
P_{3}
\end{array}\right)
$$

Fig. 5 shows the results for an austenitic stainless steel that is studied in Ref. [6]. The values of the interaction energies for the same austenite grain orientation are calculated using the procedure describe in Ref. [1]. As can be seen in this figure, not all 24 variants of martensite are formed. The assumption here, was that only those variants that have positive interaction energies $U>0$ can grow during transformation. This assumption was applied for the calculation of crystallographic texture of martensitic and bainitic transformation $[5,7,8]$, and correctly predict the locations where intensities in pole figures are expected. 


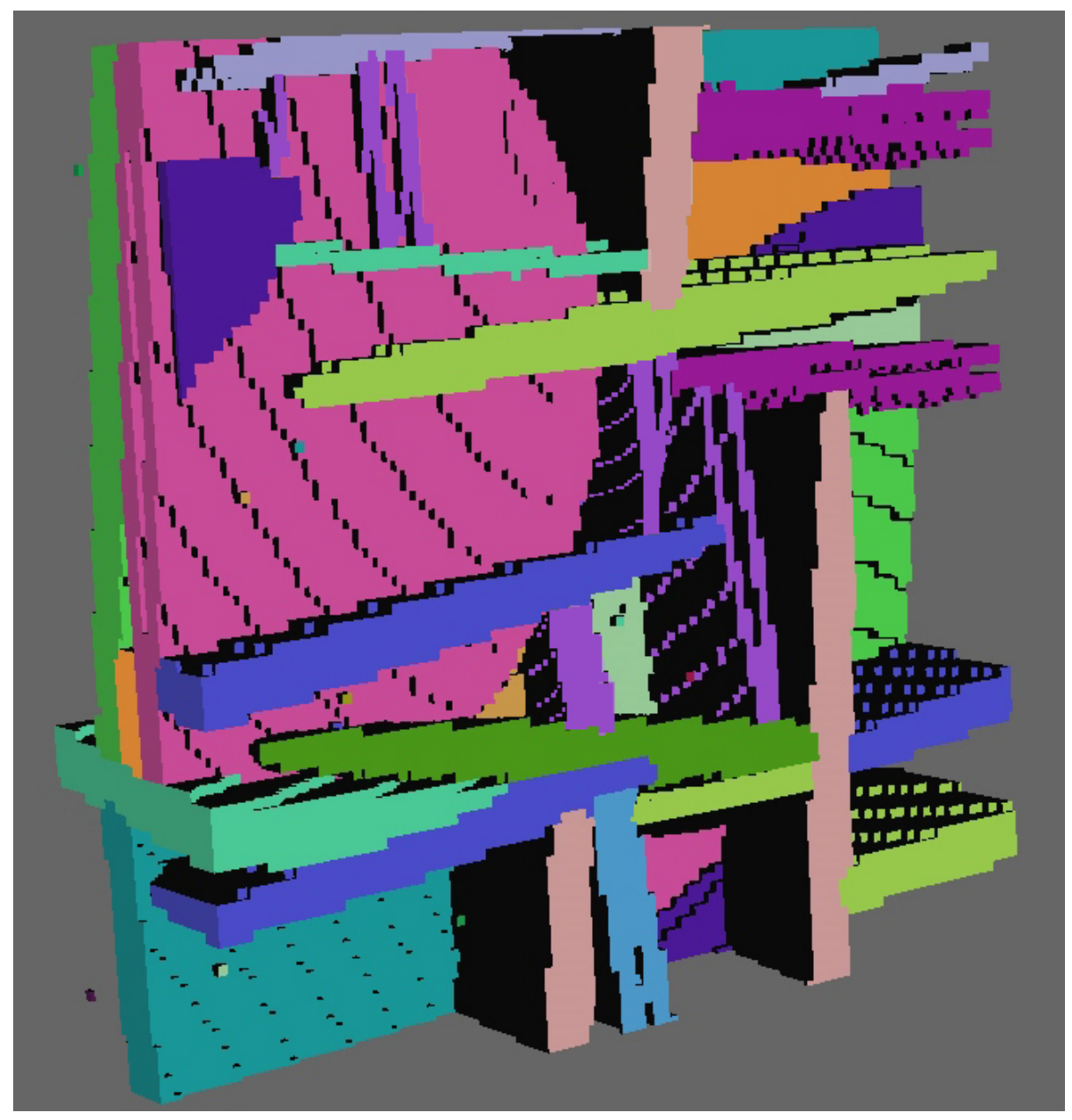

Figure 5: Missing variants due to applied external stress.

This simulation can be applied to any martensitic steel to model the 3D microstructure. However, such a computation requires both the mechanical and chemical free energies defining the martensite transformation as well as a detailed set of crystallographic data that for the martensitic transformation which includes the habit plane, the shape deformation and the orientation relationships.

\section{Conclusion}

A method is presented for modelling the three dimensional microstructure of martensite both in a transformation without an external system of applied stresses and in the presence of the external stresses. The method is based on the kinetics of crystal growth and precisely follows crystallographic theory introduced by Bowles and MacKenzi. 
Through this work, it is assumed that the materials structure is free from defects. Also, the interactions between strain fields of martensite plates are not considered in the present model. The method is programmed in $\mathrm{C}++$ and visualized with an in-house software AcaVisual. Indeed, it is able to deal with every circumstance where the shape deformation due to martensitic transformation is defined, provided that detailed crystallographic data is available.

This programme is also extended to transformation in an austenitic steel under external load, where variant selection occurs. The computer program calculates the mechanical free energy which is the interaction energy between martensite and applied stress and allows only those variants with positive $U$ to grow. This criterion was previously employed and was able to accurately predict the location of intensities in pole figure. The program is, then, able to deal with a variety of complexities including various starting austenite textures and different states of externally applied stress.

\section{Acknowledgement}

The authors are grateful for the financial support from TATA steel and the Royal Academy of Engineering of UK.

\section{References}

\section{References}

[1] Bhadeshia, H. K. D. H., 2001. Geometry of Crystals, 2nd edition, Institute of Materials.

[2] Patel, J. R., Cohen, M., 1953, Criterion for the action of applied stress in the martensitic transformation. Acta Metallurgica 1, 531-538.

[3] Bowles, J. S., MacKenzie, J. K., 1954. The crystallography of martensite transformations. Acta Metallurgica 2, 129-137.

[4] Wayman, C. M., 1964. Introduction to the Crystallography of Martensitic Transformation, MacMillan, New York.

[5] Kundu, S., Hase, K., Bhadeshia, H. K. D. H., 2007. Crystallographic texture of stress-affected bainite. Proceedings of The Royal Society A, 2309-2328.

[6] Humbert, M., Petit, B., Bolle, B., Gey, N.,2007. Analysis of the $\gamma-\epsilon-\alpha$ ' variant selection induced by $10 \%$ plastic deformation in 304 stainless steel at $-60^{\circ} \mathrm{C}$. Materials Science and Engineering A 454-455, 508-517.

[7] Kundu, S., Bhadeshia, H. K. D. H., 2007. Crystallographic texture and intervening transformation. Scripta Materialia 57, 869-872.

[8] Kundu, S., Bhadeshia, H. K. D. H., 2007. Crystallographic texture of stressaffected bainite. Proceedings of The Royal Society A 463, 2309-2328. 
[9] Falk, F., 1983. One-dimensional model of shape memory alloys. Archives of Mechanics $35,63-84$.

[10] Barsch, G.R., Krumhansl, J.A., 1984. Twin boundaries in ferroelastic media without interface dislocations. Physical Review Letters 53, 1069-1072.

[11] Barsch, G.R., Krumhansl, J.A., 1984. Nonlinear and nonlocal continuum model of transformation precursors in martensites. Metallurgical Transactions A 19, 761-775.

[12] Saxena, A., Wu, Y., Lookman, T., Shenoy, S.R., Bishop, A.R., 1997. Hierarchical pattern formation in elastic materials, Physica A: Statistical Mechanics and its Applications 239, 18-34.

[13] Rasmussen, K.O., Lookman, T., Saxena, A., Bishop, A.R., Albers, R.C., Shenoy, S.R., 2001. Three-dimensional elastic compatibility and varieties of twins in martensites. Physical Review Letters 87, 055704-1-055704-4.

[14] Ahluwalia, R., Lookman, T., Saxena, A., Albers, R.C., 2004. Landau theory for shape memory polycrystals. Acta Materialia 52, 209-218.

[15] Ahluwalia, R., Lookman, T., Saxena, 2003. Elastic deformation of polycrystals. Physical Review Letters 91, 055501.

[16] Cui, Y., W., Koyama, T., Ohnuma, I., Oikawa, K., Kainuma, R., Ishida, K., 2007. Simulation of hexagonal-orthorhombic phase transformation in polycrystals. Acta Materialia 2007, 233-241.

[17] Shchyglo, O., Salman, U., Finel, A., 2012. Martensitic phase transformations in Ni-Ti-based shape memory alloys: The Landau theory. Acta Materialia 60, 67846792.

[18] Wang, Y., Khachaturyan, A.G., 1997. Three-dimensional field model and computer modelling of martensitic transformations. Acta Materialia 45, 759-773.

[19] Li, D.Y., Chen, L.Q., 1997. Shape of a rhombohedral coherent $T i_{11} N i_{14}$ precipitate in a cubic matrix and its growth and dissolution during constrained aging. Acta Materialia 45, 2435-2442.

[20] Artemev, A., Jin, Y., Khachaturyan, A.G., 2001. Three-dimensional phase field model of proper martensitic transformation. Acta Materialia 2001, 1165-1177.

[21] Artemev, A., Wang, Y., Khachaturyan, A.G., 2000. Three-dimensional phase field model and simulation of martensitic transformation in multilayer systems under applied stresses. Acta Materialia 2000, 2503-2518.

[22] Jin, Y.M., Artemev, A., Khachaturyan, A.G., 2001. Three-dimensional phase field model of low-symmetry martensitic transformation in polycrystal: simulation of martensite in AuCd alloys. Acta Materialia 49, 2309-2320. 
[23] Yamanaka, A., Takaki, T., Tomita, Y., 2008. Elastoplastic phase filed model of self and plastic accomadations in cubic to tetragonal martensitic transformation. Materials Science and engineering A 491, 378-384.

[24] Levitas, V. I., Preston, D. L., 2002. Three-dimensional Landau theory for multivariant stress-induced martensitic phase transformations. I. Austenite $\longleftrightarrow$ martensite. Physical Review B 66, 134206.

[25] Levitas, V. I., Preston, D. L., 2002. Three-dimensional Landau theory for multivariant stress-induced martensitic phase transformations. II. Multivariant phase transformations and stress space analysis. Physical Review B 66, 134207.

[26] Levitas, V. I., Preston, D. L., 2002. Three-dimensional Landau theory for multivariant stress-induced martensitic phase transformations. III. Alternative potentials, critical nuclei, kink solutions, and dislocation theory. Physical Review B 66, 134201.

[27] MacKenzie, J. K., Bowles, J. S., 1954. The crystallography of martensite transformation. Acta Metallurgic 2, 138-147.

[28] Wechsler, M S., Lieberman, D. S., Read, T. A., 1953. On the theory of the formation of martensite. Trans. AIME Journal of Metals 197, 1503-1515.

[29] Koistinen, D. P., Marburger, R. E., 1959. A general equation prescribing the extent of the austenite-martensite transformation in pure iron-carbon alloys and plain carbon steels.Acta Metalurgica 7, 59-60.

[30] Bhadeshia, H., Strang, A., Gooch, D.J., 1998. Ferritic power plant steels: remanent life assessment and approach to equilibrium. International Meterials Review 43, 45-69.

[31] Speer, J., Matlock, D.K., De Cooman, B.C., Schroth, J.G., 2003. Carbon partitioning into austenite after martensite transformation. Acta Materialia 51, 26112622.

[32] Bhadeshia, H. K. D. H., Honeycombe, R. W. K., 2006. Steels microstructure and properties, 3rd edition, Elsevier Ltd.

[33] Harris, W. J., Cohen, M., 1949. Transaction of American Institute of Mining (Metallurgical) Engineering 180, 447-470.

[34] Olson, G. B., Cohen, M., 1975. Kinetics of Strain-Induced Martensitic Nucleation, Metallurgical Transaction 6A. 791-795.

[35] Raghavan, V., Cohen, M., 1971. Measurement and Interpretation of Isothermal Martensitic Kinetics, Metallurgical Transactions 2. 2409-2418.

[36] Gao, Q., Wang, C., Qu, F., Wang, Y., Qiao, Z., 2014. Martensite transformation kinetics in $9 \mathrm{Cr}-1.7 \mathrm{~W}-0.4 \mathrm{Mo}-\mathrm{Co}$ ferritic steel. Journal of Alloys and Compounds $610,322-330$. 
[37] Bate, P., Hutchinson, B., 2000. The effect of elastic interactions between displacive transformations on texture in steels. Acta Materialia 48, 3183-3192.

[38] Gey, N., Petit, B., Humbert, M., 2005. Electron backscattered diffraction study of $\varepsilon / \alpha$ ' martensitic variants induced by plastic deformation in 304 stainless steel. Metallurgical and Materials Transactions A 36A, 3291-3299.

[39] Stewart, J. W., Thomson, R. C., Bhadeshia, H. K. D. H., 1994. Cementite precipitation during tempering of martensite under the influence of an externally applied stress. Journal of Materials science 29, 6079-6084. 